\title{
SOME DIFFERENTIAL AND ALGEBRAIC CONSEQUENCES OF THE EINSTEIN FIELD EQUATIONS*
}

\author{
BY \\ K. W. LAMSON
}

Introduction. If a set of four directions in a Riemannian four-dimensional space, $V_{4}$, is orthogonal, then the $d s^{2}$ can be expressed in terms of their sixteen parameters, $h_{k}^{\alpha}\left(x_{0}, x_{1}, x_{2}, x_{3}\right)$, as in Einstein's recent papers.

The first purpose of this paper is to set up sixteen invariant linear firstorder partial differential equations in these parameters ( $\$ 2)$. The solutions of these equations include all solutions for empty space of the Einstein field equations of 1917. There is a restriction which excludes some special cases. In addition to the $h_{k}^{\alpha}$, these sixteen equations contain four linear combinations of the components of the curvature tensor. These four combinations are to be taken as independent variables, $x^{k}$.

Since only alternating tensors appear it is convenient to use Cartan's notation $\dagger$ for symbolic differential forms and for their derivatives and products. Covariant differentiation in the sense of the absolute differential calculus is not used, except in $\S 4$.

The components of the curvature tensor may be taken as coefficients in the equation of a quadratic line complex in a three-dimensional projective space, $P_{3} \ddagger \S$. The directions $h_{k}{ }^{\alpha}$ correspond to the vertices of a tetrahedron in $P_{3}$. Bivector and simple bivector correspond to linear complex and special complex. Where there is no danger of misunderstanding, the language of $V_{4}$ will be used interchangeably with that of $P_{3}$. The second purpose of this paper is the application of some of the theory of quadratic complexes to the study of the curvatures in $V_{4} \cdot$.

The lines which lie in a plane in $P_{3}$ and which belong to the quadratic complex are tangent to a conic. The envelope of planes for which this conic

\footnotetext{
* Presented to the Society, December 27, 1923, and September 12, 1930; received by the editors February 20, 1930.

$\dagger$ Goursat, Leçons sur le Problème de Pfaff, chapters I and III.

$\ddagger \mathrm{D}$. J. Struik, On sets of principal directions in a Riemannian manifold in four dimensions, Journal of Mathematics and Physics of the Massachusetts Institute of Technology, vol. 7 (1928), p. 193.

E. Kretschmann, Annalen der Physik, vol. 53 (1917), p. 592.

I Jessop, Treatise on the Line Complex. See also Hudson, Kummer's Quartic Surface, and Zindler's article in the Encyclopedia, IIIC8.
} 
becomes two plane pencils is a Kummer surface. For the tropes of this surface the two vertices of these pencils coincide in a point which lies on the surface. Each of the sixteen tropes is tangent to the surface along a conic.

Quadratic complexes were classified by Klein in types characterized by the elementary divisors of their quadratic forms taken with that of the Plücker identity. In this paper the type [111111] is assumed throughout.

In $\S 6, \theta$-functions are introduced as a further application of the theory of complexes, and also for reasons of symmetry. No one of the ten quadrics or of the many tetrahedra associated with the curvature tensor is held out against the others.

1. Notation. The differential of length is the sum of the squares of four Pfaff expressions,

$$
\begin{aligned}
\omega_{k} & =h_{k \mid \alpha} d x^{\alpha} \\
d s^{2} & =\omega_{0}{ }^{2}+\omega_{1}{ }^{2}+\omega_{2}{ }^{2}+\omega_{3}{ }^{2} .
\end{aligned} \quad(k=0,1,2,3),
$$

The Ricci coefficients of rotation are given by

$$
2 \gamma_{i j k}=h_{k \mid \alpha}\left(h_{i \rho}^{\alpha} h_{j}^{\rho}-h_{j \rho}^{\alpha} h_{i}^{\rho}\right)+h_{j \mid \alpha}\left(h_{i \rho}^{\alpha} h_{k}^{\rho}-h_{k \rho}^{\alpha} h_{i}^{\rho}\right)+h_{i \mid \alpha}\left(h_{k \rho}^{\alpha} h_{j}^{\rho}-h_{j \rho}^{\alpha} h_{k}^{\rho}\right),
$$

where the matrix $h_{k}^{\alpha}$ is the reciprocal of $h_{k \mid \alpha}$, and the subscript $\rho$ denotes ordinary differentiation.

The components of the curvature tensor are $\gamma_{i j, k l}$, referred to the directions $h_{k}^{\alpha}$, found by putting three of the $\omega_{k}$ equal to zero.* Greek and Roman indices are used when the axis-system is given by $d x^{\alpha}=0$ and $\omega_{k}=0$, respectively.

The symbolic product of two Pfaffians is illustrated by

$$
\left[\omega_{1} \omega_{2}\right]=h_{1 \mid \alpha} h_{2 \mid \beta}\left(d x^{\alpha} \delta x^{\beta}-d x^{\beta} \delta x^{\alpha}\right) .
$$

Symbolic forms of the second and third degree are linear combinations of

$$
d x^{\alpha} \delta x^{\beta}-d x^{\beta} \delta x^{\alpha}
$$

or

$d x^{\alpha} \delta x^{\beta} \Delta x^{\gamma}+d x^{\beta} \delta x^{\gamma} \Delta x^{\alpha}+d x^{\gamma} \delta x^{\alpha} \Delta x^{\beta}-d x^{\alpha} \delta x^{\gamma} \Delta x^{\beta}-d x^{\beta} \delta x^{\alpha} \Delta x^{\gamma}-d x^{\gamma} \delta x^{\beta} \Delta x^{\alpha}$.

If two forms have a common factor of the first degree their product is zero. When the square of a form is written, ordinary multiplication is meant.

* Levi-Civita, A Simplified Presentation of Einstein's Unified Field Equations, p. 6, formula 8; Vereinfachte Herstellung der Einstein'schen einheitlichen Feldgleichungen, Sitzungsberichte der Preussischen Akademie der Wissenschaften, 1929, p. 139, formula (8). 
The derivative of a form of degree $n$ is a form of degree $n+1$, is indicated by a prime, and illustrated by

$$
\omega_{k}^{\prime}=\gamma_{k j i}\left[\omega_{i} \omega_{i}\right] .
$$

In $V_{4}$ the $\Omega_{k}$ are defined as follows in terms of symbolic products of the $\omega_{k}$. In $P_{3}$ the definitions in terms of the Plücker line coördinates are used:

$$
\begin{aligned}
& \Omega_{1}=\left[\omega_{0} \omega_{1}\right]+\left[\omega_{2} \omega_{3}\right]=p_{01}+p_{23}, \\
& \Omega_{2}=\left[\omega_{0} \omega_{2}\right]+\left[\omega_{3} \omega_{1}\right]=p_{02}+p_{31}, \\
& \Omega_{3}=\left[\omega_{0} \omega_{3}\right]+\left[\omega_{1} \omega_{2}\right]=p_{03}+p_{12} ; \\
& \bar{\Omega}_{1}=-\left[\omega_{0} \omega_{1}\right]+\left[\omega_{2} \omega_{3}\right]=-p_{01}+p_{23}, \\
& \bar{\Omega}_{2}=-\left[\omega_{0} \omega_{2}\right]+\left[\omega_{3} \omega_{1}\right]=-p_{02}+p_{31}, \\
& \bar{\Omega}_{3}=-\left[\omega_{0} \omega_{3}\right]+\left[\omega_{1} \omega_{2}\right]=-p_{03}+p_{12} .
\end{aligned}
$$

These expressions equated to zero are the six fundamental complexes of Klein.

The following equations define the Pfaff expressions $\mu_{k}$ and $\bar{\mu}_{k}$ :

$$
\begin{array}{ll}
\mu_{1}=\left(\gamma_{01 k}+\gamma_{23 k}\right) \omega_{k}, & \bar{\mu}_{1}=\left(-\gamma_{01 k}+\gamma_{23 k}\right) \omega_{k}, \\
\mu_{2}=\left(\gamma_{02 k}+\gamma_{31 k}\right) \omega_{k}, & \bar{\mu}_{2}=\left(-\gamma_{02 k}+\gamma_{31 k}\right) \omega_{k}, \\
\mu_{3}=\left(\gamma_{03 k}+\gamma_{12 k}\right) \omega_{k}, & \bar{\mu}_{3}=\left(-\gamma_{03 k}+\gamma_{12 k}\right) \omega_{k} .
\end{array}
$$

Then, from (1.1) and (1.2),

$$
\begin{array}{ll}
\Omega_{1}^{\prime}=\left[\Omega_{2} \mu_{3}\right]-\left[\Omega_{3} \mu_{2}\right], & \bar{\Omega}_{1}^{\prime}=\left[\bar{\Omega}_{2} \bar{\mu}_{3}\right]-\left[\bar{\Omega}_{3} \bar{\mu}_{2}\right], \\
\Omega_{2}^{\prime}=\left[\Omega_{3} \mu_{1}\right]-\left[\Omega_{1} \mu_{3}\right], & \bar{\Omega}_{2}^{\prime}=\left[\bar{\Omega}_{3} \bar{\mu}_{1}\right]-\left[\bar{\Omega}_{1} \bar{\mu}_{3}\right], \\
\Omega_{3}^{\prime}=\left[\Omega_{1} \mu_{2}\right]-\left[\Omega_{2} \mu_{1}\right], & \bar{\Omega}_{3}^{\prime}=\left[\bar{\Omega}_{1} \bar{\mu}_{2}\right]-\left[\bar{\Omega}_{2} \bar{\mu}_{1}\right] .
\end{array}
$$

To the hypercone of minimal directions in $V_{4}$ corresponds a quadric surface in $P_{3}$, which will be referred to as $d s^{2}$.

2. First-order differential equations. For the $d s^{2}$ of $\$ 1$ the Einstein field equations for empty space can be written as follows:

$$
\begin{aligned}
\gamma_{01,01}-\gamma_{23,23} & =0 \\
\gamma_{02,02}-\gamma_{31,31} & =0 \\
\gamma_{03,03}-\gamma_{12,12} & =0 \\
\gamma_{02,03}+\gamma_{31,03}-\gamma_{02,12}-\gamma_{31,12} & =0 \\
\gamma_{02,03}-\gamma_{31,03}+\gamma_{02,12}-\gamma_{31,12} & =0 \\
\gamma_{03,01}+\gamma_{12,01}-\gamma_{03,23}-\gamma_{12,23} & =0
\end{aligned}
$$




$$
\begin{aligned}
& \gamma_{03,01}-\gamma_{12,01}+\gamma_{03,23}-\gamma_{12,23}=0, \\
& \gamma_{01,02}+\gamma_{23,02}-\gamma_{01,31}-\gamma_{23,31}=0, \\
& \gamma_{01,02}-\gamma_{23,02}+\gamma_{01,31}-\gamma_{23,31}=0 .
\end{aligned}
$$

A consequence of these nine is the tenth equation

$$
\gamma_{01,01}+\gamma_{02,02}+\gamma_{03,03}=\text { const. (the cosmological } \lambda \text { ). }
$$

The nine equations can be written as the following three equations in symbolic differential forms of the second degree, each equivalent to six scalar equations:*

$$
\begin{aligned}
& \mu_{1}^{\prime}+\left[\mu_{2} \mu_{3}\right]=A_{11} \Omega_{1}+A_{12} \Omega_{2}+A_{13} \Omega_{3}, \\
& \mu_{2}^{\prime}+\left[\mu_{3} \mu_{1}\right]=A_{21} \Omega_{1}+A_{22} \Omega_{2}+A_{23} \Omega_{3}, \\
& \mu_{3}^{\prime}+\left[\mu_{1} \mu_{2}\right]=A_{31} \Omega_{1}+A_{32} \Omega_{2}+A_{33} \Omega_{3} \quad\left(A_{j k}=A_{k j}\right) .
\end{aligned}
$$

Symbolic multiplication by $\bar{\Omega}_{1}, \bar{\Omega}_{2}, \bar{\Omega}_{3}$ gives the equations (2.1) to $(2.9)$ as indicated in the following table.

$$
\begin{array}{l|lll}
\multicolumn{1}{c}{} & (2.11) & (2.12) & (2.13) \\
\cline { 2 - 4 } \bar{\Omega}_{1} & (2.1) & (2.9) & (2.6) \\
\bar{\Omega}_{2} & (2.8) & (2.2) & (2.5) \\
\bar{\Omega}_{3} & (2.7) & (2.4) & (2.3)
\end{array}
$$

The $A_{j k}$ are undetermined coefficients which drop out for these multiplications, because the complexes $\Omega$ are in involution, or in other words, because the symbolic product of any two different ones is zero.

Another way of writing the field equations is as follows:

$$
\begin{aligned}
& \bar{\mu}_{1}^{\prime}+\left[\bar{\mu}_{2} \bar{\mu}_{3}\right]=\bar{A}_{11} \bar{\Omega}_{1}+\bar{A}_{12} \bar{\Omega}_{2}+\bar{A}_{13} \bar{\Omega}_{3}, \\
& \bar{\mu}_{2}^{\prime}+\left[\bar{\mu}_{3} \bar{\mu}_{1}\right]=\bar{A}_{21} \bar{\Omega}_{1}+\bar{A}_{22} \bar{\Omega}_{2}+\bar{A}_{23} \bar{\Omega}_{3}, \\
& \bar{\mu}_{3}^{\prime}+\left[\bar{\mu}_{1} \bar{\mu}_{2}\right]=\bar{A}_{31} \bar{\Omega}_{1}+\bar{A}_{32} \bar{\Omega}_{2}+\bar{A}_{33} \bar{\Omega}_{3} \quad\left(\bar{A}_{j k}=\bar{A}_{k j}\right) .
\end{aligned}
$$

The quadratic complex whose coefficients are the components of the curvature tensor is then

$$
\begin{aligned}
& A_{11} \Omega_{1}{ }^{2}+A_{22} \Omega_{2}{ }^{2}+A_{33} \Omega_{3}{ }^{2}+\bar{A}_{11} \bar{\Omega}_{1}^{2}+\bar{A}_{22} \bar{\Omega}_{2}{ }^{2}+\bar{A}_{33} \bar{\Omega}_{3}{ }^{2} \\
+ & 2\left(A_{23} \Omega_{2} \Omega_{3}+A_{31} \Omega_{3} \Omega_{1}+A_{12} \Omega_{1} \Omega_{2}+\bar{A}_{23} \bar{\Omega}_{2} \bar{\Omega}_{3}+\bar{A}_{31} \bar{\Omega}_{3} \bar{\Omega}_{1}+\bar{A}_{12} \bar{\Omega}_{1} \bar{\Omega}_{2}\right)=0 .
\end{aligned}
$$

The products are ordinary, not symbolic.

If the complex is of type [111111], it has associated with it six fundamen-

* Cartan, Equations de la Gravitation d'Einstein, Journal de Mathématiques, (9), vol. 1 (1922), p. 153. 
tal linear complexes.* From the way in which these are found it follows from (2.17) that the field equations for empty space are the necessary and sufficient condition that the three linear complexes of one regulus of $d s^{2}$ be linear combinations of three of the fundamental complexes, and that the complexes of the other regulus be combinations of the remaining three. If the complex is not of the above type this statement may require modification.

By multiplying (2.11) symbolically with $\Omega_{1}$, and (2.12) with $\Omega_{3}$ (or (2.13) with $\Omega_{2}$ ) it is seen that

and similarly

$$
\begin{aligned}
& 2 A_{11}=\gamma_{01,01}+2 \gamma_{01,23}+\gamma_{23,23} \quad(\text { cyclic } 1,2,3), \\
& 2 A_{23}=\gamma_{02,03}+\gamma_{31,03}+\gamma_{02,12}+\gamma_{31,12} \quad(\text { cyclic } 1,2,3),
\end{aligned}
$$

$$
\begin{aligned}
& 2 \bar{A}_{11}=\gamma_{01,01}-2 \gamma_{01,23}+\gamma_{23,23} \quad(\text { cyclic } 1,2,3), \\
& 2 \bar{A}_{23}=\gamma_{02,03}-\gamma_{31,03}-\gamma_{02,12}+\gamma_{31,12} \quad(\text { cyclic } 1,2,3) .
\end{aligned}
$$

So far there are nine independent equations in the sixteen unknowns, $h_{k}{ }^{\alpha}$. For the purposes of this section there are added the six equations

$$
A_{j k}=\bar{A}_{j k}=0 \quad(j \neq k) .
$$

This reduces the quadratic complex to the Klein form, and amounts to taking the axes of reference in $V_{4}$ as directions which are cut by the $P_{3}$ in any one of those six fundamental tetrahedra which are self-polar with respect to $d s^{2}$. In fact the field equations in the case [111111] are satisfied if and only if there are six tetrahedra which simultaneously reduce the $d s^{2}$ to a sum of squares of Pfaffians and the quadratic complex to the Klein form.

Differentiating (2.11), in the sense of Cartan, and using (2.18), we get

$$
\left[\mu_{2}^{\prime} \mu_{3}\right]-\left[\mu_{3}^{\prime} \mu_{2}\right]=A_{11} \Omega_{1}^{\prime}+\left[A_{11}^{\prime} \Omega_{1}\right] \text {. }
$$

Substituting for $\mu_{2}^{\prime}, \mu_{3}^{\prime}$, and $\Omega_{1}^{\prime}$ from (2.12), (2.13), and (1.3), we get the four scalar equations included in the third-degree symbolic equation

$$
A_{22}\left[\Omega_{2} \mu_{3}\right]-A_{33}\left[\Omega_{3} \mu_{2}\right]=A_{11}\left(\left[\Omega_{2} \mu_{3}\right]-\left[\Omega_{3} \mu_{2}\right]\right)+\left[A_{11}{ }^{\prime} \Omega_{2}\right],
$$

and also get five other sets of four equations from (2.12) to (2.16).

These equations written in full are as follows:

$$
\begin{aligned}
& h_{0}^{\alpha} \partial A_{11} / \partial x^{\alpha}=\left(A_{33}-A_{11}\right)\left(\gamma_{022}+\gamma_{312}\right)-\left(A_{11}-A_{22}\right)\left(\gamma_{033}+\gamma_{123}\right), \\
& h_{1}^{\alpha} \partial A_{11} / \partial x^{\alpha}=\left(A_{33}-A_{11}\right)\left(-\gamma_{023}-\gamma_{313}\right)-\left(A_{11}-A_{22}\right)\left(\gamma_{032}+\gamma_{122}\right), \\
& h_{2}^{\alpha} \partial A_{11} / \partial x^{\alpha}=\left(A_{33}-A_{11}\right)\left(-\gamma_{020}-\gamma_{310}\right)-\left(A_{11}-A_{22}\right)\left(-\gamma_{031}-\gamma_{121}\right), \\
& h_{3}^{\alpha} \partial A_{11} / \partial x^{\alpha}=\left(A_{33}-A_{11}\right)\left(\gamma_{021}+\gamma_{311}\right)-\left(A_{11}-A_{22}\right)\left(-\gamma_{030}-\gamma_{120}\right)
\end{aligned}
$$

\footnotetext{
* Jessop, loc. cit., p. 189, and Klein, Mathematische Annalen, vol. 2 (1870), p. 198.
} 


$$
\text { In (2.19) the indices }(1,2,3) \text { are replaced with }(2,3,1) \text {; }
$$

(2.21) In (2.19) the indices $(1,2,3)$ are replaced with $(3,1,2)$;

$$
\begin{aligned}
& h_{0}^{\alpha} \partial \bar{A}_{11} / \partial x^{\alpha}=\left(\bar{A}_{33}-\bar{A}_{11}\right)\left(\gamma_{022}-\gamma_{312}\right)-\left(\bar{A}_{11}-\bar{A}_{22}\right)\left(\gamma_{033}-\gamma_{123}\right), \\
& h_{1}^{\alpha} \partial \bar{A}_{11} / \partial x^{\alpha}=\left(\bar{A}_{33}-\bar{A}_{11}\right)\left(\gamma_{023}-\gamma_{313}\right)-\left(\bar{A}_{11}-\bar{A}_{22}\right)\left(-\gamma_{032}+\gamma_{122}\right), \\
& h_{2}^{\alpha} \partial \bar{A}_{11} / \partial x^{\alpha}=\left(\bar{A}_{33}-\bar{A}_{11}\right)\left(-\gamma_{620}+\gamma_{310}\right)-\left(\bar{A}_{11}-\bar{A}_{22}\right)\left(\gamma_{031}-\gamma_{121}\right), \\
& h_{3}^{\alpha} \partial \bar{A}_{11} / \partial x^{\alpha}=\left(\bar{A}_{33}-\bar{A}_{11}\right)\left(-\gamma_{021}+\gamma_{311}\right)-\left(\bar{A}_{11}-\bar{A}_{22}\right)\left(-\gamma_{030}+\gamma_{120}\right) ;
\end{aligned}
$$

(2.23) In (2.21) the indices $(1,2,3)$ are replaced with $(2,3,1)$;

(2.24) In (2.21) the indices $(1,2,3)$ are replaced with $(3,1,2)$.

The addition of (2.19), (2.20), and (2.21) shows that

$$
A_{11}+A_{22}+A_{33}=\text { const. (the cosmological } \lambda \text { ), }
$$

and similarly

$$
\bar{A}_{11}+\bar{A}_{22}+\bar{A}_{33}=\lambda \text {. }
$$

There remain sixteen independent equations, for example (2.20), (2.21), (2.23), and (2.24). If four of the $A$ 's are independent, which is to be expected in the general case, they may be taken as independent variables. The main result of this section may be expressed as follows.

Every solution of the Einstein field equations in empty space, of type[111111], and with four independent components of the curvature, is included (up to change of variable) among the solutions of the linear first-order equations(2.19) to (2.24), where

$$
\begin{array}{ll}
A_{11}=\lambda-x^{0}-x^{1}, & \bar{A}_{11}=\lambda-x^{2}-x^{3}, \\
A_{22}=x^{0}, & \bar{A}_{22}=x^{2}, \\
A_{33}=x^{1}, & \bar{A}_{33}=x^{3},
\end{array}
$$

and the $\gamma_{i j k}$ are the Ricci coefficients of rotation.

If there are any solutions of this type for which the $A$ 's are not independent, the elimination of the $A$ 's would lead to algebraic equations in the coefficients of rotation.

Conversely it can be shown by differentiation that a consequence of (2.19) etc. is

$$
A_{j k}=\bar{A}_{j k}=0 \quad(j \neq k) .
$$

3. Characteristic properties of the complex for the case $\lambda=0$. As before, it is possible to impose six conditions on the twelve $A$ 's appearing in (2.11) 
to (2.16). Suppose that this is done so that they are given by the following combinations of six new functions $b_{1}, b_{2}, b_{3}, a_{11}, a_{22}, a_{33}$ :

$$
\begin{array}{lrl}
A_{j k} & =2 b_{j} b_{k}+a_{j k} & (j, k=1,2,3) ; \\
\bar{A}_{j k}=a_{j k} & \left(a_{j k}=0, j \neq k\right) .
\end{array}
$$

The quadratic complex is then referred to a Rosenhain tetrahedron of nodes and tropes, found by taking the polar planes of any node in $\bar{\Omega}_{1}, \bar{\Omega}_{2}, \bar{\Omega}_{3}$, and the poles of these planes with respect to the same complexes.*

Its equation in Plücker coördinates is

$$
\begin{aligned}
{\left[b_{1}\left(p_{01}+p_{23}\right)\right.} & \left.+b_{2}\left(p_{02}+p_{31}\right)+b_{3}\left(p_{03}+p_{12}\right)\right]^{2} \\
& +2 a_{11} p_{01} p_{23}+2 a_{22} p_{02} p_{31}+2 a_{33} p_{03} p_{12}=\gamma_{i j, k l} p_{i j} p_{k l}=0
\end{aligned}
$$

being given by adding the squared equation of a linear complex to that of a tetrahedral complex. If $\lambda=0$, it follows from (2.10) that

$$
b_{1}^{2}+b_{2}^{2}+b_{3}^{2}=0 \text {. }
$$

Therefore the linear complex is special (the bivector is simple) and its directrix is one of the lines of that regulus of $d s^{2}$ which is given by

$$
\bar{\Omega}_{1}=\bar{\Omega}_{2}=\bar{\Omega}_{3}=0 .
$$

In Hudson's notation (p. 78) the vertices of the tetrahedron chosen above are the nodes corresponding to four dots in a row. Four dots in a column might have been used. This would lead to a line in the other regulus.

It is characteristic of the case $\lambda=0$ that the eight linear complexes associated with the eight Rosenhain tetrahedra of nodes self-polar with respect to ds ${ }^{2}$ are special. Their lines are rulings of $d s^{2}$, four to each regulus.

The rest of this section is devoted to formulas for these lines. There is a principal tangent on the Kummer surface whose tangent lines are secondorder singular lines of the complex. $\dagger$ Let $\left(r_{0}, r_{1}, r_{2}, r_{3}\right)$ be the coördinates, referred to a fundamental tetrahedron, of the one point not a node where this curve touches the conic in one of the tropes $\left(t_{0}, t_{1}, t_{2}, t_{3}\right)$. Then $(r)$ is also the plane tangent to the same principal tangent through the node which is the pole of that trope in $d s^{2}$. For the node $(t)$ the complex cone degenerates into the pencil of lines in the plane $(r)$ through the point $(t)$. For the trope $(t)$ the complex conic degenerates into the pencil in the plane $(t)$ through the point $(\boldsymbol{r})$.

The four nodes (tropes) of the Rosenhain tetrahedron and the planes

* Jessop, loc. cit., p. 157, and Hudson, loc. cit., p. 83.

† Jessop, loc. cit., p. 95. 
(points) of the corresponding pencils as just described are given in the following table.

Nodes (tropes) referred to a Corresponding planes (points) referred to tetrahedron which is

a tetrahedron which is

Fundamental Rosenhain Fundamental Rosenhain

$t_{0}, \quad t_{1}, \quad t_{2}, \quad t_{3} \quad 1,0,0,0 \quad r_{0}, \quad r_{1}, \quad r_{2}, \quad r_{3} \quad 0, \quad b_{1}, \quad b_{2}, \quad b_{3}$ $-t_{1}, \quad t_{0}, \quad t_{3},-t_{2} \quad 0,1,0,0-r_{1}, \quad r_{0}, \quad r_{3},-r_{2}-b_{1}, \quad 0, \quad b_{3},-b_{2}$ $-t_{2},-t_{3}, \quad t_{0}, \quad t_{1} \quad 0,0,1,0-r_{2},-r_{3}, \quad r_{0}, \quad r_{1}-b_{2},-b_{3}, \quad 0, \quad b_{1}$ $-t_{3}, \quad t_{2},-t_{1}, \quad t_{0} \quad 0,0,0,1-r_{3}, \quad r_{2},-r_{1}, \quad r_{0}-b_{3}, \quad b_{2},-b_{1}, \quad 0$

From the usual formulas for the incidence of line and plane and from (3.2) it is seen that the four planes of the last set contain the directrix of the special complex $(b)$. Therefore the determinant of the third set is zero and of rank two. The resulting equation is

$$
r_{0}^{2}+r_{1}^{2}+r_{2}^{2}+r_{3}^{2}=0
$$

which states that the point (plane) ( $r$ ) lies on (is tangent to) the quadric $d s^{2}$. In the following table the four points in any row or column lie on a line. These eight lines are those referred to in the above italicized statement.

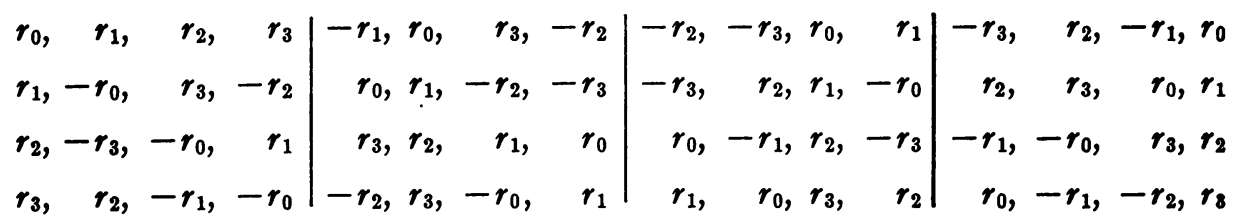

In computing this result it is convenient to have the $\gamma_{i j, k l}$ or $A_{j k}$ expressed in terms of the $r_{k}$ and $t_{k}$. Let the coefficient of proportionality of the $t_{k}$ be chosen so that

$$
\sum t_{k}^{2}=1 \text {. }
$$

Then the required expressions are obtained from the analytic statement that the complex cone through $(t)$ is the plane $(r)$ taken twice. All the $\gamma_{i j, k l}$ can be obtained from the following, which also give the factor of proportionality of the $r_{k}$ :

$$
\begin{aligned}
& \gamma_{01,01}+\gamma_{02,31}-\gamma_{03,12}=-\left(r_{0} r_{1}+r_{2} r_{3}\right) /\left(t_{0} t_{1}+t_{2} t_{3}\right) \quad(\text { cyclic 1, 2, 3); } \\
& \gamma_{01,01}-\gamma_{02,31}+\gamma_{03,12}=-\left(-r_{0} r_{1}+r_{2} r_{3}\right) /\left(-t_{0} t_{1}+t_{2} t_{3}\right)(\text { cyclic } 1,2,3) \\
& r_{0}{ }^{2}=\gamma_{01,01} t_{1}{ }^{2}+\gamma_{02,02} t_{2}{ }^{2}+\gamma_{03,03} t_{3}{ }^{2}, \\
& r_{1}^{2}=\gamma_{01,01} t_{0}{ }^{2}+\gamma_{02,02} t_{3}{ }^{2}+\gamma_{03,03} t_{2}{ }^{2} \text {, } \\
& r_{2}^{2}=\gamma_{01,01} t_{3}^{2}+\gamma_{02,02} t_{0}^{2}+\gamma_{03,03} t_{1}^{2} \text {, } \\
& r_{3}{ }^{2}=\gamma_{01,001} t_{2}{ }^{2}+\gamma_{02,02} t_{1}{ }^{2}+\gamma_{03,03} t_{0}{ }^{2} .
\end{aligned}
$$


No use was made of $\lambda=0$ in the derivation of equations (3.4), (3.5), and (3.6), and they are true if $\lambda \neq 0$.

The addition of the four equations (3.6) and substitution from (2.10) give

$$
r_{0}^{2}+r_{1}^{2}+r_{2}^{2}+r_{3}^{2}=\lambda .
$$

The complex cone for each of the sixteen points $(r)$ in the tropes as described above, degenerates into the plane $(t)$ which contains that $(r)$, and another plane $(w)$. The coördinates of $(w)$ are readily obtained from its definition. Then the differences between the cases $\lambda=0$ and $\lambda \neq 0$ are given in the following table of incidences. The entries "yes" and "no" indicate whether there is incidence or not.

$$
\begin{aligned}
& \lambda=0 \quad \lambda \neq 0 \\
& \text { Planes Plames }
\end{aligned}
$$

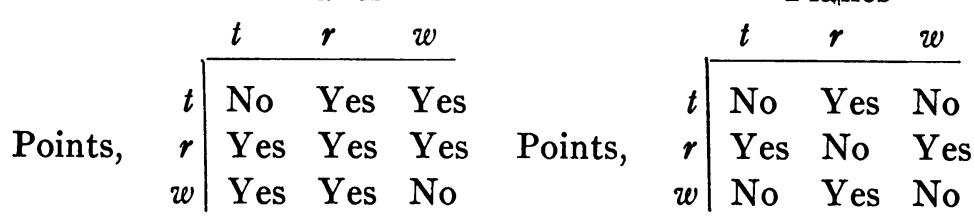

If $\lambda=0$, and not otherwise, the point (w) lies on the conic in the trope $(t)$, and is in fact the other point besides $(r)$ where the plane $(r)$ cuts the conic. Also, in this case only, the line joining $(r)$ and $(w)$ is tangent to $d s^{2}$.

It is characteristic of the case $\lambda=0$ that the point $(r)$ which lies on the conic also lies on the quadric $d s^{2}$. Since there are four such points, there are four complexes in the co-singular family for which $\lambda=0 . \dagger$

4. Singular lines of the quadratic complex. If a vector is displaced parallel to itself around an infinitesimal parallelogram it will not in general return to its original position. In this section the cases where it does so return are described in terms of the singular lines of the complex. $\ddagger$ The Einstein equations and a fundamental tetrahedron of reference are assumed with either $\lambda=0$ or $\lambda \neq 0$. The vector difference between the vector in its final and initial positions is given by

$$
\Delta v^{j}=\gamma_{i j, k l} \omega_{l} \omega^{*}{ }_{k} v^{i}=\frac{1}{2} \gamma_{i j, k l} p_{l k} v^{i},
$$

where $\omega_{k}$ and $\omega_{k}{ }^{*}$ are the differentials determining the parallelogram. If there is a $v^{k}$ for which $\Delta v^{k}$ vanishes for a given $p_{k l}$, then the determinant of

† Jessop, loc. cit., chapter 8 .

$\ddagger$ Jessop, loc. cit., p. 90. 
coefficients of $v^{k}$ is zero, and in fact of rank two. The point $v^{k}$ can lie anywhere on a certain line $\left(s_{k l}\right)$. The condition on $\left(p_{k l}\right)$ turns out to be

$$
A_{11}^{2}\left(p_{01}+p_{23}\right)^{2}-\bar{A}_{11}^{2}\left(-p_{01}+p_{23}\right)^{2}+(\text { cyclic } 1,2,3)=0 .
$$

The Plücker coördinates of the line $(s)$ are

$$
s_{01}=\gamma_{01,01} p_{23}+\gamma_{01,23} p_{01}, \quad s_{23}=\gamma_{01,01} p_{01}+\gamma_{01,23} p_{23}
$$

(cyclic 1, 2, 3).

If the line $(p)$ cuts the line $(s)$ then

$$
A_{11}\left(p_{01}+p_{23}\right)^{2}+\bar{A}_{11}\left(-p_{01}+p_{23}\right)^{2}+(\text { cyclic } 1,2,3)=0
$$

which is the same as the original complex $\gamma_{i j, k l} p_{i j} p_{k l}=0$. Equations (4.2) and (4.3) state that $(p)$ is a singular line of the first order. If in addition ( $s)$ belongs to the complex (4.3), then

$$
A_{11}^{8}\left(p_{01}+p_{23}\right)^{2}+\bar{A}_{11}^{3}\left(-p_{01}+p_{23}\right)^{2}+(\text { cyclic } 1,2,3)=0,
$$

and $(p)$ is a singular line of the second order.

5. Through a point in any $V_{4}$ there are two-spread elements having the property that if any vector in the element is displaced around a closed path in the element, then the increment of the vector will lie in the element. In the relativity case for empty space there are twenty-six of these. In $P_{3}$ the Plücker coördinates of the corresponding lines referred to a fundamental tetrahedron are given in the following table:

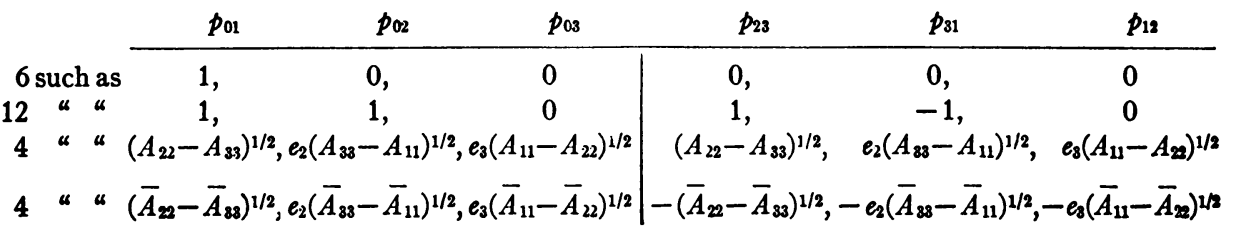

where $e_{2}$ and $e_{3}$ are either +1 or -1 . The first eighteen listed above are the edges of the six fundamental tetrahedra which are self-polar with respect to $d s^{2}$, and the other eight are rulings of $d s^{2}$. This table was computed from (4.1) and from the equations which state that $v^{k}$ and $\Delta v^{k}$ lie on $p_{j k}$.

6. $\theta$-functions. This section contains formulas for the components of the curvature in terms of $\theta$-functions of two variables. The coördinates of points on the Kummer surface are expressed uniformly by means of these functions.* The branch points of the corresponding Riemann surface are $A_{11}, A_{22}, A_{33},-\bar{A}_{11},-\bar{A}_{22},-\bar{A}_{33}$, defined in $\S 2$, with $A_{j k}=0$ for $j \neq k$. Using $\sum r_{k} t_{k}=0$, the equations (3.4) and (3.5) may be written

* Encyklopädie der Mathematischen Wissenschaften, II B7, p. 747. Humbert, Journal de Mathématiques, (4), vol. 9 (1893), p. 29. Hudson, loc. cit., p. 181. This reference is probably the most convenient in this connection. 
(6.1) $\gamma_{01,01}+\gamma_{02,31}-\gamma_{03,12}=-\left({\overline{\Omega_{2, r t}}}^{2}-\Omega_{1, r t}^{2}-\Omega_{2, r t}^{2}+q_{1}^{2}\right) / Q_{1}^{2}$,

(6.2) $\gamma_{01,01}-\gamma_{02,31}+\gamma_{03,12}=-\left(\Omega_{2, r t}^{2}-\bar{\Omega}_{1, r t}^{2}-\bar{\Omega}_{2, r t}^{2}+\bar{q}_{1}^{2}\right) / \bar{Q}_{1}^{2}$,

where

$$
\begin{array}{rlrl}
\Omega_{1, r t} & =r_{1} t_{0}-r_{0} t_{1}+r_{3} t_{2}-r_{2} t_{3} & & (\text { cyclic } 1,2,3), \\
\bar{\Omega}_{1, r t} & =-r_{1} t_{0}+r_{0} t_{1}+r_{3} t_{2}-r_{2} t_{3} & & (\text { cyclic } 1,2,3), \\
q_{1} & =r_{1} t_{0}+r_{0} t_{1}+r_{3} t_{2}+r_{2} t_{3}, & \\
\bar{q}_{1} & =-r_{1} t_{0}-r_{0} t_{1}+r_{3} t_{2}+r_{2} t_{3}, & \\
Q_{1}=2\left(t_{0} t_{1}\right. & \left.+t_{2} t_{3}\right), \quad \bar{Q}_{1}=2\left(-t_{0} t_{1}+t_{2} t_{3}\right) .
\end{array}
$$

The expressions appearing on the right hand sides are the bilinear and quadratic forms discussed in Hudson.* The quadratic forms $Q_{1}$ and $\bar{Q}_{1}$ with the eight other similar ones are the squares of $\theta$-functions with zero arguments, with a factor of proportionality $k_{1}$. Let $u=\left(u_{1}, u_{2}\right)$ be the parameters of the point $r_{k}$. Since $(r)$ is on the conic in trope $(t)$, the $\theta$ with zero characteristic vanishes, $\theta_{0}(u)=0$. Then the bilinear forms in $t_{k}$ and $r_{k}$ are the squares of the thetas with argument $(u), \dagger$ with a factor of proportionality $k_{2}$. Hudson's notation for the characteristics (p. 178) will be used. The zero characteristic is denoted by the symbol $d d$ or 0 .

The factor $k_{1}$ is given by

$$
1=k_{1} \theta_{d d}^{2}(0) \text {. }
$$

If $\lambda \neq 0$, the factor $k_{2}$ may be found as follows. The sum of the squares of the bilinear forms appearing in any row or column of the table in Hudson (p. 30) is found to be $\sum r^{2}$ which by (3.7) is equal to $\lambda$. Thus

$$
k_{2}^{2}\left[\theta_{d d}^{4}(u)+\theta_{a b}^{4}(u)+\theta_{b c}^{4}(u)+\theta_{c a}^{4}(u)\right]=\lambda .
$$

Replacing the bracket by its equivalent in terms of a theta with doubled argument $\ddagger$

$$
k_{2}^{2} \theta_{d d}^{3}(0) \theta_{d d}(2 u)=\lambda
$$

Equation (6.1) then becomes

$$
\begin{aligned}
\gamma_{01,01}+\gamma_{02,31}-\gamma_{03,12}= & -k_{2}^{2}\left[\theta_{b a}^{4}(u)-\theta_{b c}^{4}(u)\right. \\
& \left.-\theta_{a b}^{4}(u)+\theta_{a d}^{4}(u)\right] /\left\{k_{1}^{2} \theta_{a d}^{4}(0)\right\} .
\end{aligned}
$$

* Loc. cit., p. 30.

$\dagger$ Hudson, loc. cit., p. 180.

‡ Schleiermacher, Mathematische Annalen, vol. 50 (1898), p. 200, formula 4. 
This bracket also may be simplified by using the thetas with doubled argument. In the Riemann theta formula, ${ }^{*}$ put the characteristics $\rho=0$ and $\sigma=0$ and

$$
\begin{aligned}
u^{\prime}+v^{\prime}+w^{\prime}+t^{\prime} & =2 u, & 2 u^{\prime} & =u, \\
u^{\prime}+v^{\prime}-w^{\prime}-t^{\prime} & =0, & 2 v^{\prime} & =u, \\
u^{\prime}-v^{\prime}+w^{\prime}-t^{\prime} & =0, & 2 w^{\prime} & =u, \\
u^{\prime}-v^{\prime}-w^{\prime}+t^{\prime} & =0, & 2 t^{\prime} & =u,
\end{aligned}
$$

and the bracket becomes $\theta_{a d}^{3}(0) \theta_{a d}(2 u)$. On replacing the $k_{1}$ and $k_{2}$ by their values from (6.3) and (6.4), equation (6.5) becomes

$$
\gamma_{01,01}+\gamma_{02,31}-\gamma_{03,12}=-\frac{\lambda \theta_{d d}(0)}{\theta_{d d}(2 u)} \cdot \frac{\theta_{a d}(2 u)}{\theta_{a d}(0)} .
$$

Thus the desired expressions for the components of the curvature tensor in the case $\lambda \neq 0$ are given in the following table of ten equations; each of the sixteen elements in the first matrix is equal to the corresponding element in the second with the indicated factor:

$$
\begin{aligned}
& \begin{array}{||cccc}
-\lambda, & 0, & 0, & 0 \\
0, & \lambda-2 \gamma_{01,01}, & \gamma_{03,03}-\gamma_{01,23}+\gamma_{02,31}, & \gamma_{02,02}+\gamma_{03,12}-\gamma_{01,23} \\
0, & \gamma_{03,03}+\gamma_{01,23}-\gamma_{02,31}, & \lambda-2 \gamma_{02,02}, & \gamma_{01,01}-\gamma_{02,31}+\gamma_{03,12} \\
0, & \gamma_{02,02}-\gamma_{03,12}+\gamma_{01,23}, & \gamma_{01,01}+\gamma_{02,31}-\gamma_{03,12}, & \lambda-2 \gamma_{03,03}
\end{array} \|
\end{aligned}
$$

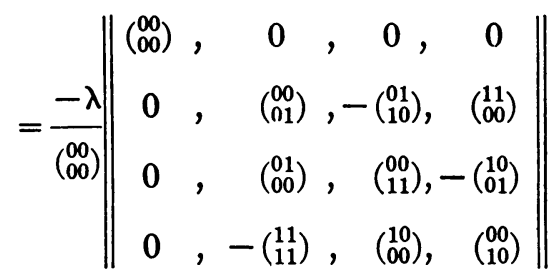

with

$$
\gamma_{i j, i k}=0 \quad(j \neq k) ; \quad \gamma_{01,01}=\gamma_{23,23} \quad(\text { cyclic } 1,2,3)
$$

where the symbol $\left(\begin{array}{c}\alpha \beta \\ \gamma_{\delta}\end{array}\right)$ denotes the quotient

$$
\theta\left[\begin{array}{c}
\alpha \beta \\
\gamma_{\delta}
\end{array}\right](2 u) / \theta\left[\begin{array}{c}
\alpha \beta \\
\gamma \delta
\end{array}\right](0) .
$$

The derivation of the other equations in the table is similar to that of (6.6). If $\lambda=0$, the point $(r)$ whose parameters are $(u)$ lies on $d s^{2}$, and therefore $\theta_{d d}(2 u)=0 . \dagger$ The same equations are true in this case, with a different fac-

* Krazer, Theorie der Zweifach Unendlichen Thetareihen, pp. 2, 9 and 65 (the case 8-10-14+2). See also the Encyclopedia article above, p. 673.

† Hudson, loc. cit., p. 189. 
tor of proportionality. This factor cannot be found in terms of the $\gamma_{i j, k l}$ at a single point in $V_{4}$.

7. Reality relations. In the formula

$$
d s^{2}=\omega_{0}^{2}+\omega_{1}^{2}+\omega_{2}^{2}+\omega_{3}^{2},
$$

$\omega_{0}$ is a pure imaginary and the other $\omega_{k}$ are real, for real values of the $x^{\alpha}$ and $d x^{\alpha}$. This convention of signs, while not the usual one, is used so that the writers on the line complex may be more closely followed. The coefficients of rotation $\gamma_{i j k}$ and the curvatures $\gamma_{i j, k l}$ are real if the index 0 occurs an even number of times, and are otherwise pure imaginary. In the preceding sections the bar over a letter has meant a different function. In the physical case the bar is to mean conjugate imaginary.*

A point in $P_{3}$, or vector in $V_{4}$, is real if the ratio of the $j$ th coördinate $(j=1,2,3)$ to the 0 th is pure imaginary. From (3.1) and (3.2) it can be shown that each of the four points $(r)$ in the four real tropes is real. These are the points on the main diagonal of the table before (3.4). Only one of the six fundamental tetrahedra self-polar with respect to $d s^{2}$ has four real vertices.

If the $\theta$-functions are defined as in Hudson (p. 176) and in Baker's book on Abelian functions (p. 248),

$$
\theta\left[\begin{array}{c}
\alpha_{1}, \alpha_{2} \\
\beta_{1}, \beta_{2}
\end{array}\right](u)=\sum \exp \left\{2 \pi i\left(n+\frac{1}{2} \alpha\right)\left(u+\frac{1}{2} \beta\right)+\pi i \tau\left(n+\frac{1}{2} \alpha\right)^{2}\right\},
$$

where $\alpha, \beta, n$ and $u$ are row letters and $\tau$ is a two-by-two matrix, then $\tau_{11}$ is the negative conjugate of $\tau_{22}$, and $\tau_{12}$ is pure imaginary. kind:

This is now proved by considering the hyperelliptic integrals of the first

$$
\begin{gathered}
w_{1}=\int \frac{d z}{R}, \quad w_{2}=\int \frac{z d z}{R}, \\
R=\left[\left(A_{11}-z\right)\left(-\bar{A}_{11}-z\right)\left(A_{22}-z\right)\left(-\bar{A}_{22}-z\right)\left(A_{33}-z\right)\left(-\bar{A}_{33}-z\right)\right]^{1 / 2} .
\end{gathered}
$$

The six branch points are situated symmetrically with respect to the axis of imaginaries. This follows from the expressions for the $A_{j j}$ and $\bar{A}_{i j}$ in $\$ 2$, and from the reality criterion near the beginning of this section. In constructing the Riemann surface take, for example, the $a_{1}$ and $b_{1}$ cuts $\uparrow$ wholly in the first and fourth quadrants. Then take the $a_{2}$ and $b_{2}$ cuts symmetrically in the second and third. The moduli of periodicity are seen to have the reality relations given by the following table:

* The situation is discussed in Hudson's book on p. 126, under the case IVa.

† See Goursat-Appell, Théorie des Fonctions Algêbriques et de leurs Intégrales, Chapter III, pp. $132,133,151$ for notation and details. 


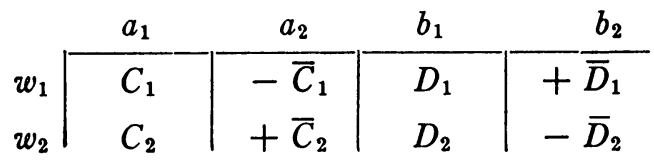

When the integrals are normalized in the usual way the required result is obtained. From this result and from the definition of the thetas it follows that $\theta_{d d}(0)$ is real, and then that the ten non-vanishing thetas with zero argument, when arranged as in $\$ 6$, form an Hermitian matrix.

8. The Schwarzschild solution. This does not come under the preceding discussion because the complex is not of type [111111]. In fact if $x_{0}$ is the time, and $x_{1}$ is taken radially from the central mass, then

$$
A_{11}=\bar{A}_{11}=-\frac{2 m}{x_{1}{ }^{3}}, \quad A_{22}=\bar{A}_{22}=A_{33}=\bar{A}_{33}=\frac{m}{x_{1}{ }^{3}} .
$$

The complex is of type [(11)(11)11] and is a special case of the harmonic complex.*

The detailed examination of the complex (2.7) shows that the field equations exclude the cases of elementary divisors listed below. All except these twelve are algebraically possible:

$$
\begin{array}{lllll}
{[(22)],} & {[(411)],} & {[(42)],} & {[(51)],} & {[6],} \\
{[(22) 2],} & {[4(11)],} & {[42],} & {[51],} & \\
{[222],} & {[(41) 1],} & & \\
& {[411] .} & &
\end{array}
$$

* Jessop, loc. cit., pp. 209, and 111.

LEHIGH UNIVERSITY,

Bethlehex. Pa. 